\title{
The development of the internal pyramidal layer neurons of the brain parietal cortex in rats after prenatal alcohol
} exposure

\author{
Sergey M Zimatkin* and Lizaveta I Bon \\ Grodno State Medical University, Grodno, Belarus
}

\begin{abstract}
The aim of the paper was to estimate histologically the effect of alcohol consumption by rats during pregnancy on the parietal cortex neurons development in their offspring. Female Wistar rats consumed a $15 \%$ solution of ethanol as a single source of drinking $(3.64 \pm 2.2 \mathrm{~g} / \mathrm{kg} / \mathrm{day})$ throughout pregnancy, control rats received equivolume amounts of water. The offspring were decapitated on the 2-, 5-, 10-, 20-, 45-, and $90^{\text {th }}$ days after birth and the samples of the brain parietal cortex were prepared for histological examination in combination with morphometry to examine the $5^{\text {th }}$ layer inner pyramidal neurons.

Results: Antenatal alcohol exposure in rats increased (on the $2^{\text {nd }}$ and $5^{\text {th }}$ postnatal days), and then reduced (on the $10^{\text {th }}$ and $90^{\text {th }}$ days) the parietal cortex thickness, decreased the amount of parietal cortex inner pyramidal neurons and increased the number of their pathological forms at all time intervals of the examination. Starting from the $20^{\text {th }}$ postnatal day the shrinkage and cessation of the growth of inner pyramidal neurons was observed.

Conclusion: Alcohol consumption by rats during pregnancy induces deep and long-term histological changes in the parietal cortex neurons in postnatal ontogenesis in rat offspring including early swelling and postpone shrinkage and the cessation of growth of the brain cortex inner pyramidal neurons.
\end{abstract}

\section{Introduction}

It is well known that the nervous system, especially the brain, is particularly sensitive to prenatal alcohol exposure. The latter proved to induce various severe mental and behavioral disorders in children and adults, violations in the intellectual sphere, impairment of attention, memory and other cognitive functions, disturbances in the emotional sphere, impaired social interaction, sensomotor activity, preference to alcohol both in humans and experimental animals [1-4]. Magnetic resonance imaging (MRI) following prenatal alcohol exposure revealed the significant changes of neurovisual parameters of the brain including the thickening of the brain and cerebellum cortex, diffuse white matter disorders, increase in the volume of the ventricles, anomalies of the corpus callosum, significant reduction of subcortical nuclei. The severity and prevalence of them in the brain structures depend greatly on the dose and duration of alcohol exposure and the time of the study $[5,6]$.

In our previous papers we described the dynamics of histological, ultrastructural and histochemical changes of the frontal cortex $5^{\text {th }}$ layer neurons in rats subjected to antenatal exposure to alcohol $[7,4]$.

The aim of the present study is to estimate the effect of prenatal alcohol exposure on histological morphometric characteristics of parietal cortex inner pyramidal neurons in rats at different time periods after birth.

\section{Materials and methods}

\section{Animals, chemicals and experimental design}

As many as 25 female and 10 male Wistar rats were obtained from the breeding colony of the Grodno State Medical University. Their weight was $212 \pm 29$ g. All experimental procedures complied with
European Community Council Directive (86/609/EEC) for care and use of laboratory animals. Protocols were reviewed and approved by the Ethical Committee of the Grodno State Medical University. All efforts were made to minimize animal suffering. Rats were housed in vivarium with free access to standard laboratory food and kept under controlled environmental conditions. Rats of the experimental groups throughout pregnancy (from the day of detection of sperms in vaginal smears till delivery) received a $15 \%$ solution of ethanol as a single source of drinking (the average consumption of alcohol was $3.64 \pm 2.2 \mathrm{~g} / \mathrm{kg} /$ day), and the animals of the control group - equivolume amount of water. All the chemicals were obtained from Sigma-Aldrich (USA).

As many as 6 controls and 6 alcoholized rats ( 1 infant rat from each litter for every time period after birth) were decapitated on the 2-, 5-, $10-, 20-, 45-, 90^{\text {th }}$ days after birth and their brains were removed (72 samples overall total). Starting from day 45 when the sex of the animal can be distinguished, 3 male and 3 female rats from each group were elected. Samples of the parietal brain cortex were fixed in the mixture of alcohol, chloroform and acetic acid in the ratio of 6:3:1, then treated with alcohol and xilen and embedded in paraffin. The $7 \mu \mathrm{m}$ sagittal sections of the brain cortex were prepared using microtome (Leica RM2125, Germany). They were stained with $0.1 \%$ solution of thionine (the Nissl method) to assess general cytology of neurons.

Correspondence to: Sergey M. Zimatkin, Grodno State Medical University, 80 Gorkogo Street, Grodno, 230015, Belarus, Tel: (375) (152) 742492; Fax: (375) (152) 335341; E-mail: smzimatkin@mail.ru

Key words: Ethanol consumption, pregnancy, offspring, parietal cortex, neurons

Received: July 18, 2017; Accepted: August 28, 2017; Published: August 31, 2017 
For the identification of the parietal cortex in the brain sections the stereotaxic atlas was used [8]. The examination of histological preparations, their microphotography and morphometry were carried out using microscope Axioskop 2 plus (Zeiss, Germany) equipped with digital camera (Leica DFC 320, Germany) and computer image analysis software Image Warp (Bit Flow, USA). In preparations stained by the Nissl method all visible pyramidal neurons of the $5^{\text {th }}$ layer (inner pyramidal neurons) were estimated according to their type of chromatophilia (the intensity of staining of neurons cytoplasm) and divided into normochromic (normal, medium staining), hyperchromic (intense staining), hyperchromic shrunken, hypochromic (pale staining) and cell-shadows (very pale remnants of dead neurons).Three sections of the parietal cortex brain for three fields of vision for each slice were taken. Five measurements were carried out to evaluate the thickness of the parietal cortex. Ten inner pyramidal neurons of the $5^{\text {th }}$ layer of the parietal cortex was measured in every field of vision.

To estimate the size and shape of neuronal bodies the images of up to 30 neurons bodies on the computer monitor were outlined by mouse cursor. Maximal and minimal diameter $(\mathrm{D})$, perimeter $(\mathrm{P})$, square $(\mathrm{S})$, as well as form-factor $\left(4 \pi \mathrm{S} / \mathrm{P}^{2}\right.$ - parameter of sphericity and folding) and the factor of elongation (maximal D/minimal D - parameter of sphericity) were calculated.

The mean values obtained for every animal were processed with nonparametric statistics (because of the small number of animals in the groups) using software STATISTICA 10 (StatSoft, Inc., USA). In descriptive statistics, the values of median $(\mathrm{Me})$ and interquartile range (IQR) were determined. The differences were considered significant at $\mathrm{p}<0.05$ (Mann-Whitney U-test) because it was not a normal distribution

\section{Results}

Alcohol consumption by rats during pregnancy influenced the thickness of the parietal brain cortex in their offspring (Figure 1). On the $2^{\text {nd }}$ and $5^{\text {th }}$ days after birth the cortex was thicker, as compared to the controls. On the $10^{\text {th }}$ postnatal day it became significantly thinner than in the controls, on the $20^{\text {th }}$ and $45^{\text {th }}$ days the difference disappeared, but on the $90^{\text {th }}$ postnatal day it became significantly thinner again (Figure 1).

In postnatal ontogenesis (from 2 to 90 days) the density of distribution (amount per area unit) of the parietal cortex inner pyramidal neurons is regularly decreased in both control and alcohol groups (Figure 2). However, during all periods of the study in antenatally alcoholized rats a significantly lower (10-25\%) amount of neurons per unit area of the section was found (Figure 2).

In control animals, the size of the inner pyramidal parietal cortex neuron bodies increased progressively (4-fold) from the $2^{\text {nd }}$ to the $90^{\text {th }}$ postnatal day (Figure 3) In prenatally alcoholized rats a temporary increase in the area of those neurons on the $2^{\text {nd }}$ day was marked. However, starting from the $20^{\text {th }}$ postnatal day the area of the neuron bodies became significantly lower, as compared to the controls. While the size of neurons of the control animals showed continuing progressive increase, in rats exposed to alcohol prenatally following the $10^{\text {th }}$ postnatal day the neurons stopped their growing (Figures $3,4,5$ ).

In the control animals during all periods of postnatal development normochromic neurons in preparations of parietal brain cortex prevailed (60-70\%) (Figures $4,5,6)$. In prenatally alcoholized rats at all time periods of postnatal ontogenesis the number of normochromic neurons decreased significantly and the number of abnormal neurons (hyper-, hypochromic neurons and cell-shadows) increased (Figure $4,5,6)$. The greatest changes were found between the $20^{\text {nd }}$ and $90^{\text {th }}$ postnatal days. For example, on the $90^{\text {th }}$ day in the parietal cortex of prenatally alcoholized rats the amount of normochromic neurons was 2 times lower, the amount of shrunken hypochromic neurons and cellshadows was higher (by 66,20 and $40 \%$, accordingly), as compared to the controls (Figures 4, 5, 6). The amount of shrunken hyperchromic neurons in rats exposed to alcohol increased dramatically after the $10^{\text {th }}$ postnatal day and reached the maximum on the $45^{\text {th }}$ and $90^{\text {th }}$ postnatal days (Figure 7). It is associated with the changes in neurons shape: increase in their elongation and decrease in form factor (sphericity).

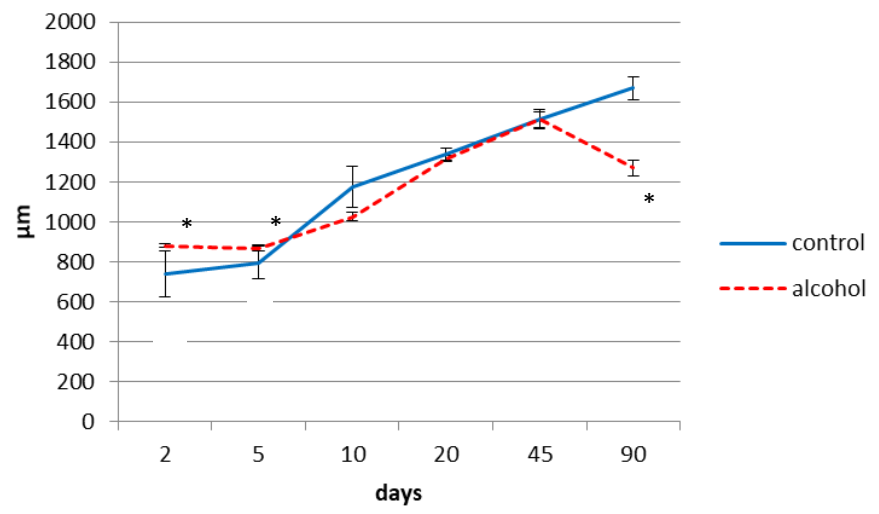

Figure 1. The thickness of the parietal cortex in rats at different time periods after birth Data are presented as median \pm interquartile range; ${ }^{*}$-p $<0.05$, as compared to the controls. Vertical bars show interquartile ranges.

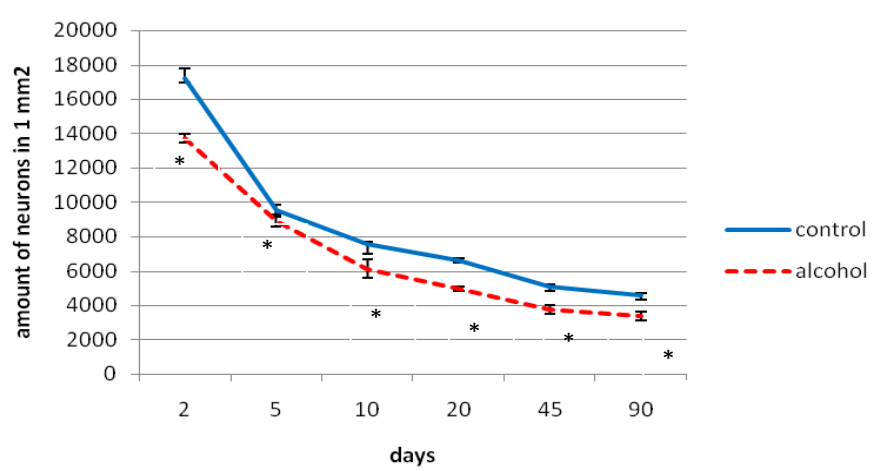

Figure 2. The density of distribution of the inner pyramidal neurons of the parietal cortex during postnatal ontogenesis. Data are presented as median \pm interquartile range; ${ }^{*}$-p $<$ 0.05 , as compared to the controls. Vertical bars show interquartile ranges.

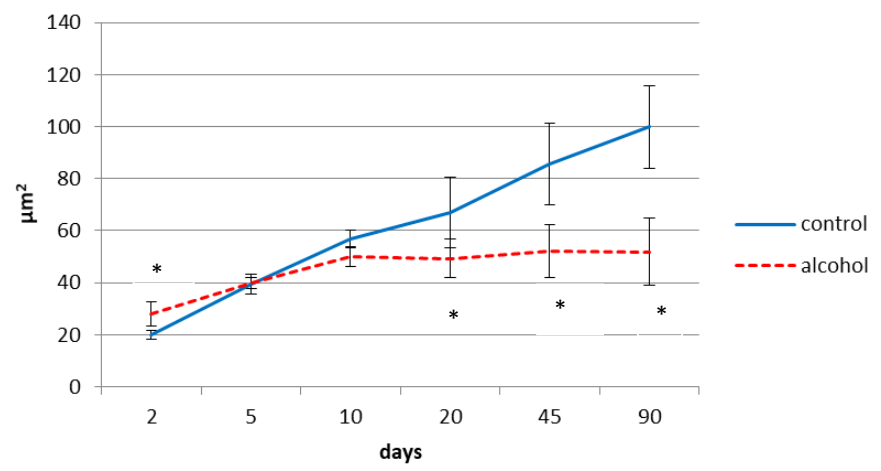

Figure 3. Area of perikaryon of the $5^{\text {th }}$ layer neurons in the parietal cortex of rats in postnatal period. Data are presented as median \pm interquartile range; ${ }^{*}$ - $p<0.05$, as compared to the controls. Vertical bars show interquartile ranges. 


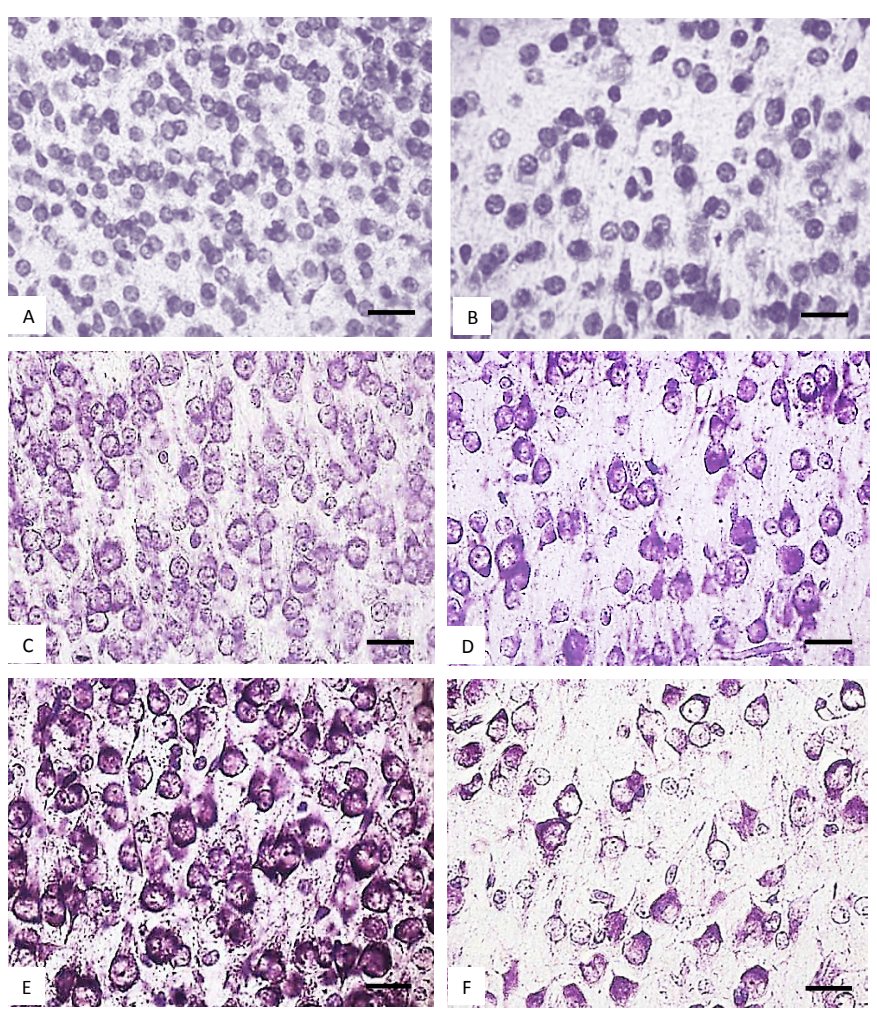

Figure 4. The parietal cortex inner pyramidal neurons $\left(5^{\text {th }}\right.$ layer) in the controls $(A, C, E)$ and antenatally alcoholized rats $(\mathrm{B}, \mathrm{D}, \mathrm{F})$ on the $2-(\mathrm{A}, \mathrm{B}), 5-(\mathrm{C}, \mathrm{D})$ and $10^{\text {th }}(\mathrm{E}, \mathrm{F})$ days after birth. Stained by the Nissl method. Digital microphotography. Scale bars $-20 \mu \mathrm{m}$, magnification-40x.

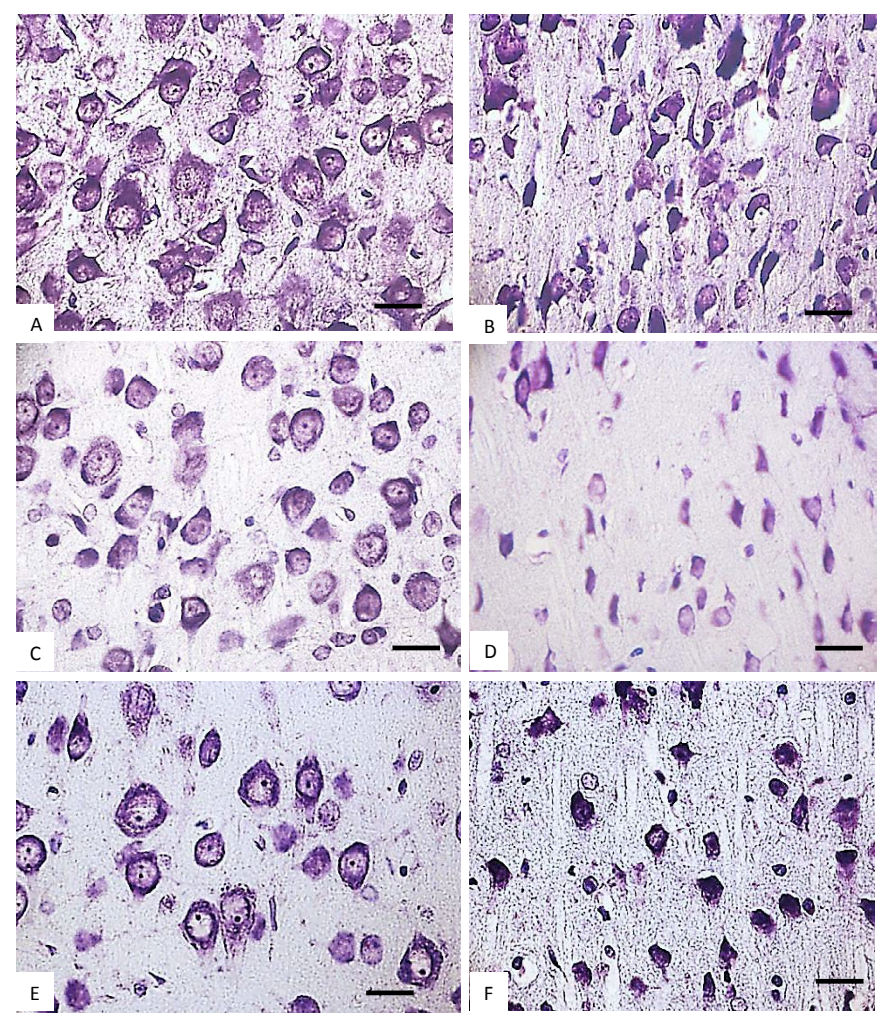

Figure 5. The parietal cortex inner pyramidal neurons ( $5^{\text {th }}$ layer) in the controls $(A, C, E)$ and antenatally alcoholized rats (B, D, F) on the 20-(A, B), 45-(C, D) and $90^{\text {th }}(\mathrm{E}, \mathrm{F})$ days after birth. Stained by the Nissl method. Digital microphotography. Scale bars $-20 \mu \mathrm{m}$, magnification-40x.
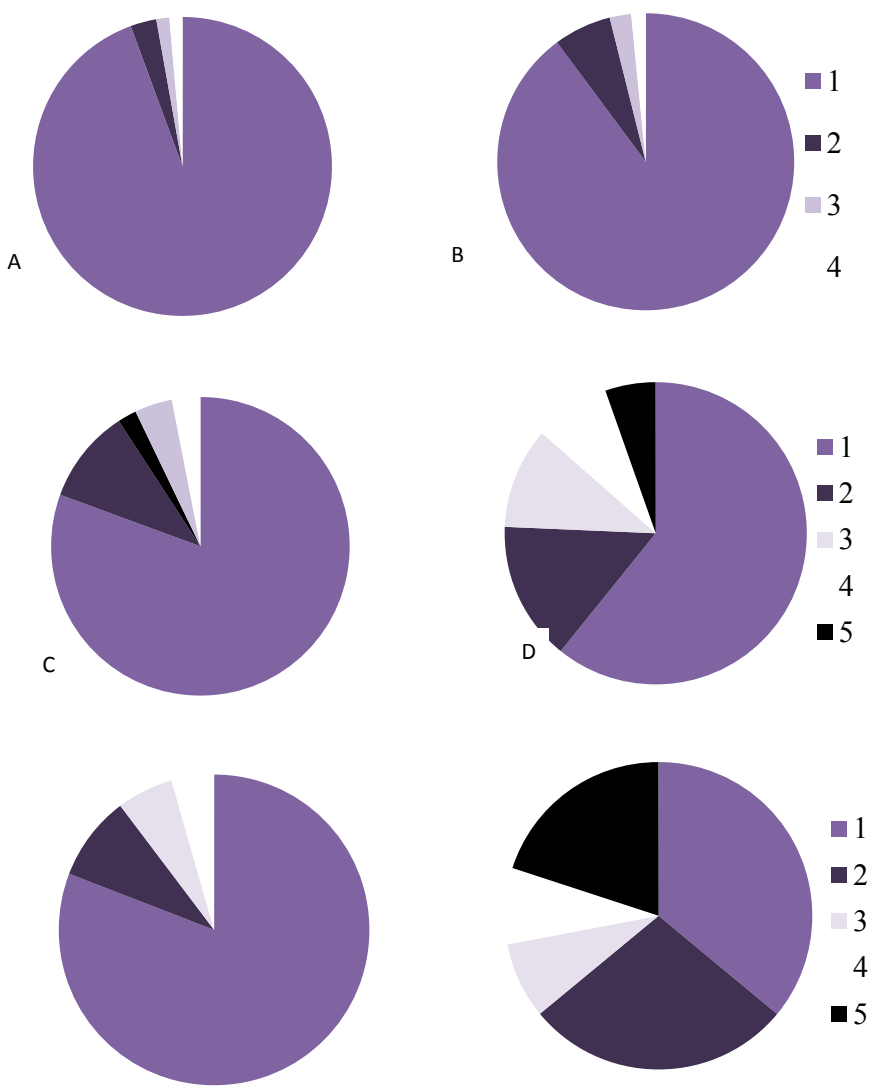

$E$

Figure 6. The percentage of neurons with different chromatophilia of cytoplasm in the parietal cortexin controls (A, C, E) and antenatally alcoholized rats (B, D, F) on the 5- (A, B), 20- $(\mathrm{C}, \mathrm{D})$ and $90^{\text {th }}(\mathrm{E}, \mathrm{F})$ days after birth

1-normochromic, 2-hyperchromic, 3-hypochromic neurons, 4-cell-shadows, 5 hyperchromic shrunken neurons.

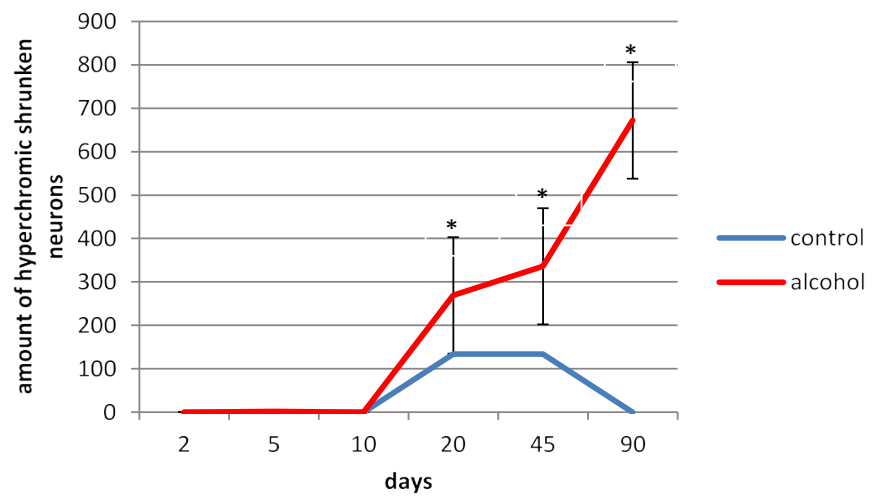

Figure 7. The amount of hyperchromic shrunken neurons in the $5^{\text {th }}$ layer parietal cortex of rats. Data presented as median \pm interquartile range; ${ }^{*}$-p $<0.05$, as compared to the controls. Vertical bars show interquartile ranges.

There is a negative correlation between the size of neurons and the number of shrunken neurons between the $20^{\text {th }}$ and $90^{\text {th }}$ days of age $(r=-$ 0.86-0.95; $\mathrm{p}<0.05)$.

\section{Discussion}

The temporary thickening of the parietal brain cortex on the $2^{\text {nd }}$ and $5^{\text {th }}$ postnatal days in the offspring of rats who consumed alcohol during pregnancy, as compared to the controls, may be associated with 
the swelling of the cortex and their neurons, which is confirmed by the pattern of perivascular edema visible in histological preparations. At that time period, the highest correlation between thickness of the cortex and area of pyramidal neurons bodies was found $(\mathrm{r}=0.79 ; \mathrm{p}<0.01)$. A late reduction of the brain cortex thickness on the $90^{\text {th }}$ postnatal day in antenatally alcoholized rats can be associated with the decrease in size and shrinkage of brain cortex neurons. There was a positive correlation between the cortex thickness and size of neurons $(r=0.77-0.94 ; \mathrm{p}<0.05)$, and a negative correlation between the cortex thickness and the amount of hyperchromic shrunken neurons $(\mathrm{r}=-0.81-0.95 ; \mathrm{p}<0.01)$ in all periods of postnatal ontogenesis in rats prenatally exposed to ethanol.

A permanently lower amount of parietal cortex neurons in all postnatal time periods studied may be due to the death of a part of neurons under the influence of alcohol during embryogenesis. Accordingly, this neurons deficiency in the brain cortex is permanent throughout postnatal development. As it is known, the weight of the brain cortex in the offspring of rats exposed to alcohol during pregnancy is reduced, it becomes thinner and contains fewer neurons and glia (at G13 and G21) [9]. These findings contradict the results of MRI showing thickening of the brain cortex in children and adolescents with fetal alcohol syndrome [10]. Our study revealed an increased (on the $2^{\text {nd }}$ and $5^{\text {th }}$ postnatal days) and decrease (on the $90^{\text {th }}$ postnatal day) parietal cortex thickness. To explain these facts, we can assume that swelling of the gray matter which occurs following antenatal alcohol exposure is detected as the thickening of the cortex.

We observed the cessation of the growth and shrinkage of inner pyramidal parietal brain cortex neurons following the $10^{\text {th }}$ day of postnatal development. We cannot directly explain these phenomena. Antenatal alcoholization seems to break the normal program of postnatal development of the brain cortex neurons. We found the increase in the amount of abnormal forms of the surviving neurons at all study time periods. The decrease in the number of normochromic neurons and increase in the number of pathological forms of inner pyramidal neurons (hyper-, hypochromic neurons, shrunken hyperchromic neurons and cells shadows) of the parietal cortex was found. These various forms of abnormal neurons at the electron microscope level had disturbances in ultrastructure. Thus, hyperchromic neurons had much more free ribosomes, which ensures their hyperchromy by the Nissl method staining; the histochemical study demonstrates a higher content of RNA in those neurons cytoplasm [4]. This testifies the intensive biosynthesis of proteins for the own needs of neurons. Perhaps this is a way of adaptation of the brain cortex neurons to compensate the death (10-25\%) as a result of antenatal alcoholization. In hyperchromic shrunken neurons a higher electron density of hyaloplasm was found. It can be the result of dramatic loss of intracellular water [11]. Probably the shrinkage of the hyperchromic neurons may be seen as a failure of adaptation that leads to their subsequent death.

In general, the disturbances of postnatal development of inner pyramidal neurons in the parietal cortex were quite similar to those in the parietal cortex neurons of antenatally alcoholized rats we observed earlier [7]. Identified histological disturbances in the brain cortex neurons may underlie the known neurological and behavioral disorders in animals and humans after antenatal alcohol exposure [12].

In conclusion, alcohol consumption during pregnancy in rats induces deep long-term and irreversible structural disturbances in the parietal cortex inner pyramidal neurons in the offspring.

\section{Acknowledgements}

The authors are grateful to the English translator Yanina Razvodovskaya for the correction of the manuscript.

\section{Funding}

Grodno State Medical University grant for reagents and animals.

\section{Conflict of interest statement}

None declared.

\section{References}

1. Brocardo PS., Budni J, Pavesi E, Franco J (2012) Anxiety - and depression-like behaviors are accompanied by an increase in oxidative stress in a rat model of fetal alcohol spectrum disorders: Protective effects of voluntary physical exercise. Neuropharmacology 62: 16-18. [Crossref]

2. Kodituwakku PW (2009) Neurocognitive profile in children with fetal alcohol spectrum disorders. Dev Disabil Res Rev. 15: 218-224. [Crossref]

3. Shea KM, Hewitt AJ, Olmstead MC (2012) Maternal ethanol consumption. Behavior Pharmacolog. 23: 105-112. [Crossref]

4. Zimatkin SM \& Bon EI (2017) Maternal alcohol intake induced dramatic ultrastructura changes in offspring brain cortex neurons. Brain and Nerves 1: 1-4.

5. De Guio F, Mangin JF, Rivière D, Perrot M, Molteno CD, Jacobson SW, Meintjes EM, Jacobson JL (2014) A study of cortical morphology in children with fetal alcohol spectrum disorders. Hum Brain Mapp. 35: 2285-2296.

6. Parnell SE, O'Leary-Moore SK, Godin EA, Dehart DB, Johnson BW, et al. (2009) Magnetic resonance microscopy defines ethanol-induced brain abnormalities in prenatal mice: effects of acute insult on gestational day 8. Alcohol Clin Exp Res 33: 1001-1011. [Crossref]

7. Zimatkin SM \& Bon EI (2017) Dinamics of histological changes in the frontal cortex of the brain in rats subjected to antenatal exposure to alcohol. Neuroscience and Behavioral Physiology 47: 370-374.

8. Parnell SE, O'Leary-Moore SK, Godin EA, Dehart DB, Johnson BW, Allan Johnson G, Styner MA, Sulik KK (2009) Magnetic resonance microscopy defines ethanolinduced brain abnormalities in prenatal mice: effects of acute insult on gestational day 8. Alcohol Clin Exp Res. 33: 1001-1011. [Crossref]

9. Paxinos G \& Watson C (2007) The rat brain in stereotaxic coordinates (6th ed) London: Academic Press.

10. Fernández-Jaén A, Fernández-Mayoralas D (2011) A cortical thickness in fetal alcohol syndrome and attention deficit disorder. Pediatr Neurol. 45: 387-391. [Crossref]

11. Miller MW (1993) Migration of Cortical Neurons is Altered by Gestational Exposure to Ethanol. Alcoholism: Clinical and Experimental Research 17: 304-314. [Crossref]

12. Mattson SN, Riley EP (1997) Neurobehavioral and Neuroanatomical Effects of Heavy Prenatal Exposure to Alcohol. - Seattle, WA: University of Washington Press.

Copyright: (C2017 Zimatkin SM. This is an open-access article distributed under the terms of the Creative Commons Attribution License, which permits unrestricted use, distribution, and reproduction in any medium, provided the original author and source are credited. 